\title{
Identifying molecular contributors to autofluorescence of neoplastic and normal colon sections using excitation-scanning hyperspectral imaging
}

Joshua Deal

Sam Mayes

Craig Browning

Shante Hill

Paul Rider

Carole Boudreaux

Thomas C. Rich

Silas J. Leavesley 


\title{
Identifying molecular contributors to autofluorescence of neoplastic and normal colon sections using excitation-scanning hyperspectral imaging
}

\author{
Joshua Deal, ${ }^{a, b, c}$ Sam Mayes, ${ }^{\text {a,d }}$ Craig Browning, ${ }^{\text {a,d }}$ Shante Hill, ${ }^{e}$ Paul Rider, ${ }^{f}$ Carole Boudreaux, \\ Thomas C. Rich, ${ }^{\mathrm{b}, \mathrm{c}}$ and Silas J. Leavesley ${ }^{\mathrm{a}, \mathrm{b}, \mathrm{c}, \mathrm{d}, \star}$ \\ aUniversity of South Alabama, Department of Chemical and Biomolecular Engineering, Mobile, Alabama, United States \\ bUniversity of South Alabama, Department of Pharmacology, Mobile, Alabama, United States \\ cUniversity of South Alabama, Center for Lung Biology, Mobile, Alabama, United States \\ dUniversity of South Alabama, Department of Systems Engineering, Mobile, Alabama, United States \\ eUniversity of South Alabama, Department of Pathology, Mobile, Alabama, United States \\ fUniversity of South Alabama, Department of Surgery, Mobile, Alabama, United States
}

\begin{abstract}
Autofluorescence, the endogenous fluorescence present in cells and tissues, has historically been considered a nuisance in biomedical imaging. Many endogenous fluorophores, specifically, collagen, elastin, nicotinamide adenine dinucleotide, and flavin adenine dinucleotide (FAD), are found throughout the human body. In fluorescence imaging scenarios, these signals can be prohibitive as they can outcompete signals introduced for diagnostic purposes. However, autofluorescence also contains information that has diagnostic value. Recent advances in hyperspectral imaging have allowed the acquisition of significantly more data in a shorter time period by scanning the excitation spectra of fluorophores. The reduced acquisition time and increased signal-to-noise ratio allow for separation of significantly more fluorophores than previously possible. We propose to utilize excitation-scanning hyperspectral imaging of autofluorescence to differentiate neoplastic lesions from surrounding non-neoplastic "normal" tissue. The spectra of isolated autofluorescent molecules are obtained using a custom inverted microscope (TE-2000, Nikon Instruments) with an Xe arc lamp and thin-film tunable filter array (VersaChrome, Semrock, Inc.). Scans utilize excitation wavelengths from 360 to $550 \mathrm{~nm}$ in $5-\mathrm{nm}$ increments. The resultant molecule-specific spectra are used to analyze hyperspectral image stacks from normal and neoplastic colorectal tissues. Due to a limited number of samples, neoplastic tissues examined here are a pool of both colorectal adenocarcinoma and adenomatous polyps. The hyperspectral images are analyzed with ENVI software and custom MATLAB scripts, including linear spectral unmixing. Initial results indicate the ability to separate signals of endogenous fluorophores and measure the relative concentrations of fluorophores among healthy and diseased states, in this case, normal colon versus neoplastic colon. These results suggest pathology-specific changes to endogenous fluorophores can be detected using excitation-scanning hyperspectral imaging. Future work will focus on expanding the library of pure molecules, exploring histogram distance metrics as a means for identifying deviations in spectral signatures, and examining more defined disease states. () The Authors. Published by SPIE under a Creative Commons Attribution 4.0 Unported License. Distribution or reproduction of this work in whole or in part requires full attribution of the original publication, including its DOI. [DOI: 10.1117/1.JBO.24.2.021207]
\end{abstract}

Keywords: hyperspectral; fluorescence; spectroscopy; microscopy; linear spectral unmixing; autofluorescence.

Paper 180318SSRR received Jun. 1, 2018; accepted for publication Nov. 26, 2018; published online Dec. 27, 2018.

\section{Introduction}

Exogenous fluorescence labels have been used for biological imaging since the 1940s. ${ }^{1}$ Coons et al. used fluorescein chemically bound to an antibody to visualize that antibody in tissue sections, creating the field of immunofluorescence microscopy. Fluorescence microscopy advanced further with the cloning of the green fluorescent protein (GFP) in the $1990 \mathrm{~s}^{2}$ and the development of many variants of GFP. ${ }^{3}$ Combining fluorescent proteins with genetic encoding expanded the applications of fluorescence microscopy, allowing researchers to tag specific proteins with fluorescent markers and track their movements and interactions. ${ }^{4}$ A common limitation to these new fluorescence

*Address all correspondence to Silas J. Leavesley, E-mail: leavesley@ southalabama.edu techniques was the endogenous fluorescence of the cells or tissues themselves, called autofluorescence. Autofluorescent signals can compete with or overpower the signal introduced for study. This was especially true of the first fluorescent proteins, as they had yet to be optimized for imaging and had relatively weak signals. The solution was often to choose a fluorophore whose peak emission wavelength was as different as possible from the autofluorescence, alter the excitation wavelength to selectively excite the introduced fluorophore and not the autofluorescence, use a very high concentration of fluorescent label, or a combination of these approaches. ${ }^{5-7}$ These compromises often led to weak or nonspecific signals, the detection of which was further complicated by a lack of noise separation from low-sensitivity detection equipment and analysis methods. Schultz et al. ${ }^{8}$ introduced an elegant solution to separation of fluorescence signals in 2001 when he applied hyperspectral imaging to fluorescence microscopy. 
Hyperspectral imaging began with remote sensing and geologic applications by NASA. ${ }^{9,10}$ Subsequent applications of hyperspectral imaging have reached virtually every field of science, including agriculture, ${ }^{11}$ archaeology, ${ }^{12}$ astronomy, ${ }^{13}$ biomedicine,${ }^{14}$ crime scene investigation, ${ }^{15}$ environmental science, ${ }^{16}$ eye care $^{17}$ food processing, ${ }^{18}$ forensics, ${ }^{19}$ and surveillance. ${ }^{20}$ Although hyperspectral imaging was initially performed via spectral reflectance, advancements in both imaging technology and processing now allow measurement of absorbance and fluorescence. The basis of this technique is that every object interacts with light in a unique and wavelengthdependent manner, and the resultant measurements are termed as spectral signatures. The uniqueness of these signatures allows separation of each object from the bulk detected signal. ${ }^{21}$ Thus, hyperspectral imaging is especially relevant in fields, such as fluorescence microscopy, where the entire method is predicated on identifying a molecule based on its interaction with light.

While the effectiveness of signal separation provided by hyperspectral imaging clearly enhances the ability to separate autofluorescence from exogenous signals, the endogenous fluorescence itself may contain diagnostic information. Using endogenous fluorescence for diagnostic purposes is not an innovative concept, but current methodologies to investigate autofluorescent signals for medical purposes are limited in at least one of several ways: lack of distinct signal separation, low signal-to-noise ratios, or prohibitively lengthy acquisition times. ${ }^{22-25}$ Similarly, we have shown that the standard method of hyperspectral imaging microscopy, emission-scanning hyperspectral imaging, reduces detectable signal and has limited applications for time-sensitive and photosensitive studies, thereby limiting its effectiveness in medical imaging. ${ }^{26-28}$ To overcome these limitations, we have designed an excitationscanning hyperspectral imaging approach. This approach provides excitation light at discrete wavelengths over a broad wavelength range and collects all of the emitted light beyond a predetermined cutoff wavelength, which has been shown to significantly increase signal-to-noise ratio of the acquired data (10- to 100 -fold) while reducing acquisition time. ${ }^{26-28}$

Here, we evaluate excitation-scanning hyperspectral imaging for estimation of endogenous autofluorescent signals in tissues. Colorectal cancer, when skin cancers are excluded, is the third most common cancer diagnosed in men and women in the United States. The American Cancer Society estimates that 97,220 new cases of colon cancer and 43,030 new cases of rectal cancer will be diagnosed in $2018 .{ }^{29}$ An alarming development is the increasing rate of colorectal cancer in younger populations (ages 20 to 54) since the mid-2000s. ${ }^{30}$ The five-year relative survival rate for colorectal carcinoma when detected at an early stage before it has spread is $\sim 90 \%$. However, only an estimated 4 in 10 cancers are found at an early stage. ${ }^{29}$

Colorectal cancer is known to cause changes in the concentration of at least five autofluorescent molecules: collagen, elastin, flavin adenine dinucleotide (FAD), nicotinamide adenine dinucleotide (NADH), and protoporphyrin IX (PPIX). ${ }^{31-35}$ To evaluate the feasibility of excitation-scanning hyperspectral imaging for detecting these autofluorescent molecules, an excitation range of 360 to $550 \mathrm{~nm}$ was selected, and the excitation spectra of these endogenous autofluorescent molecules were measured. Spectral image data were then acquired from pairs of normal and neoplastic colorectal tissues. This approach was used to estimate the respective fluorescence contribution from autofluorescent molecules. The results suggest that excitation- scanning hyperspectral imaging may be able to identify neoplastic regions in colorectal tissue in near-real time based on changes in the spectral signature of the tissues induced by concentration changes of endogenous autofluorescent molecules.

\section{Methods}

\subsection{Tissue Specimens}

Human colon specimens were obtained from colorectal resections from the University of South Alabama Medical Center Department of Surgery under an IRB-approved protocol. All human tissue specimens were obtained as deidentified residual specimens from standard-of-care procedures. Fresh specimens were retrieved from the operating room and brought to the surgical pathology suite for assessment by the surgical pathology attending to ensure tumor adequacy for patient diagnostic purposes. If deemed adequate for harvesting additional tissue for the study, a section of the neoplasm was obtained along with a section of normal colonic tissue away from the site of the neoplasm. Both specimens were assigned the next consecutive deidentified study number and designated neoplastic versus normal colon. The section of neoplasm harvested for the study was bisected and half of the specimen was submitted for frozen section analysis to ensure adequate lesional sampling. The other half was placed in a container without fixative in a $4^{\circ} \mathrm{C}$ refrigerator for retrieval for hyperspectral imaging analysis. A frozen section was not performed on the normal colonic tissue, which was entirely submitted in a separate container without fixative and placed in a $4^{\circ} \mathrm{C}$ refrigerator for retrieval. The frozen section of the neoplasm was interpreted by a surgical pathologist, and the result of the frozen section was recorded. The final diagnosis for the neoplasm was also recorded once the patient's surgical pathology report was completed. Any variances in representation of the neoplasm between the frozen section performed for the study and the final diagnosis in the patient's report were attributed to focal sampling of the neoplasm used in this study versus extensive sampling of the neoplasm used for patient diagnosis. The pairs of neoplastic and normal colon tissues were rinsed with phosphate-buffered saline (PBS), cut into $\sim 2-\mathrm{cm}$ cubes, and imaged within $8 \mathrm{~h}$ of resection. All tissues were imaged using a $25-\mathrm{mm}$-round coverslip mounted in an AttoFluor coverslip holder (Life Technologies). Each tissue specimen imaging session included a minimum of three fields of view (FOVs), not including the FOV used to generate the background spectrum.

\subsection{Excitation-Scanning Microscope Setup and Image Acquisition}

All imaging was performed on an inverted fluorescence microscope (TE2000-U, Nikon Instruments, Melville, New York) with a $20 \times$ objective (Plan Apo $\lambda 20 \times / 0.75 \infty / 0.17$ MRD00205, Nikon Instruments) and 300-W Xe arc lamp (Titan 300, Sunoptic Technologies, Jacksonville, Florida) for excitation. Excitation wavelength tuning (360 to $550 \mathrm{~nm}$ in 5-nm increments) was achieved via a custom array of five thin-film tunable filters immediately following the excitation source (TBP01378/16, TBP01-402/16, TBP01-449/15, TBP01-501/15, and TBP01-561/14, Semrock Inc., Rochester, New York). The tunable filters were mounted in a high-speed tiltable filter-wheel (Lambda VF-5, Sutter Instrument Company). A filter cube consisting of a long-pass emission filter (BLP02 561R-25, 
Semrock Inc.) and dichroic beamsplitter (FF555-Di03, Semrock Inc.) was utilized to separate excitation from emission light at $550 \mathrm{~nm}$. An electron-multiplied charge-coupled-device (EM-CCD) camera (Rolera EM-C ${ }^{2}$, QImaging, Surrey, British Columbia) was used to acquire fluorescence images.

\subsection{Image Processing and Visualization}

Images were processed into spectral image cubes through custom MATLAB (MathWorks, Natick, Massachusetts) scripts. The resultant image data were visualized with ENVI software (Exelis Visual Information Solutions, Boulder, Colorado) as a three-dimensional image cube composed of two spatial and one spectral dimension (spectral image cube). Images were false colored in the spectral dimension according to wavelength-dependent intensity, with blue, green, and red images merged using $10 \%, 50 \%$, and $90 \%$ of the spectral range, respectively. Background subtraction and wavelength-dependent illumination were completed as described previously. ${ }^{36,37}$ Briefly, a pixel-averaged background spectrum was extracted from an FOV of each tissue specimen containing no tissue and then subtracted from all other FOVs for the respective specimens. Image stacks were corrected for wavelength-dependent illumination by multiplication of correction coefficients determined by use of a National Institute of Standards and Technology traceable lamp (LS-1-CAL-INT, Ocean Optics, Inc.) and a fiber-coupled spectrometer (QE65000, Ocean Optics, Inc.), as described previously. ${ }^{36}$

\subsection{Isolated Autofluorescence Molecule Preparation}

Suspensions of collagen, elastin, FAD, NADH, and PPIX (Sigma-Aldrich) were created from commercially available powders according to product specifications. Briefly, FAD and NADH were suspended in double distilled water, and PPIX was suspended in a 1:1 solution of dimethylformamide and methanol to achieve concentrations of $100 \mu \mathrm{M}$. A concentration of $100 \mu \mathrm{M}$ was selected to provide a high signal-to-noise ratio of the spectrum being measured while maintaining a low enough concentration to ensure that the solution was still within the linear optical response region-a standard procedure in fluorescence spectroscopy for assuring high dynamic range measurements of fluorophores without inducing fluorescence quenching (self-quenching or other nonlinear phenomena). ${ }^{38,39}$ To confirm that concentrations were within the linear optical range, a dilution response was performed for each pure autofluorescent molecule (see example in Fig. 1). Elastin was prepared as a suspension of $10 \mathrm{mg} / \mathrm{mL}$ powder suspended in $0.2 \mathrm{M}$ Tris tris (hydroxymethyl) aminomethane buffer (Tris). Collagen was prepared as a suspension of $10 \mathrm{mg} / \mathrm{mL}$ PBS with $1 \mathrm{M}$ hydrochloric acid. The resultant collagen mixture was homogenized for $1 \mathrm{~h}$. The pure samples were imaged and processed in the same manner as the tissue specimens mentioned earlier. The spectra were used to generate a spectral library for signal separation.

\subsection{Spectral Image Analysis}

Spectral images were analyzed with both ENVI software and custom MATLAB scripts. First, the processed hyperspectral image cubes were opened with ENVI software for visualization. Regions of interest (ROIs) were selected to examine spectral differences between varying structures or entire FOVs. The average spectra from each region were extracted, recorded, and averaged per FOVs, patients, and specimen types for comparison. A custom MATLAB script utilized non-negatively constrained linear regression for linear spectral unmixing (LSU) to generate a fluorescence intensity image per fluorophore in the library. Any signal not accounted for by LSU is included as the rootmean-square (RMS) error. The RMS error is calculated as the square root of the sum of the difference in measured and unmixed intensity squared divided by the sample size as follows:

RMS error $=\sqrt{\frac{\sum_{i=1}^{n}\left(m_{i}-u_{i}\right)^{2}}{n}}$,

where $m_{i}$ is the measured intensity, $u_{i}$ is the unmixed intensity, and $n$ is the sample size.

Intensity-based measurements from unmixed images were obtained using ImageJ software (National Institutes of Health, Bethesda, Maryland) and compared with each other and to the total fluorescence signal available in the image. Specifically,

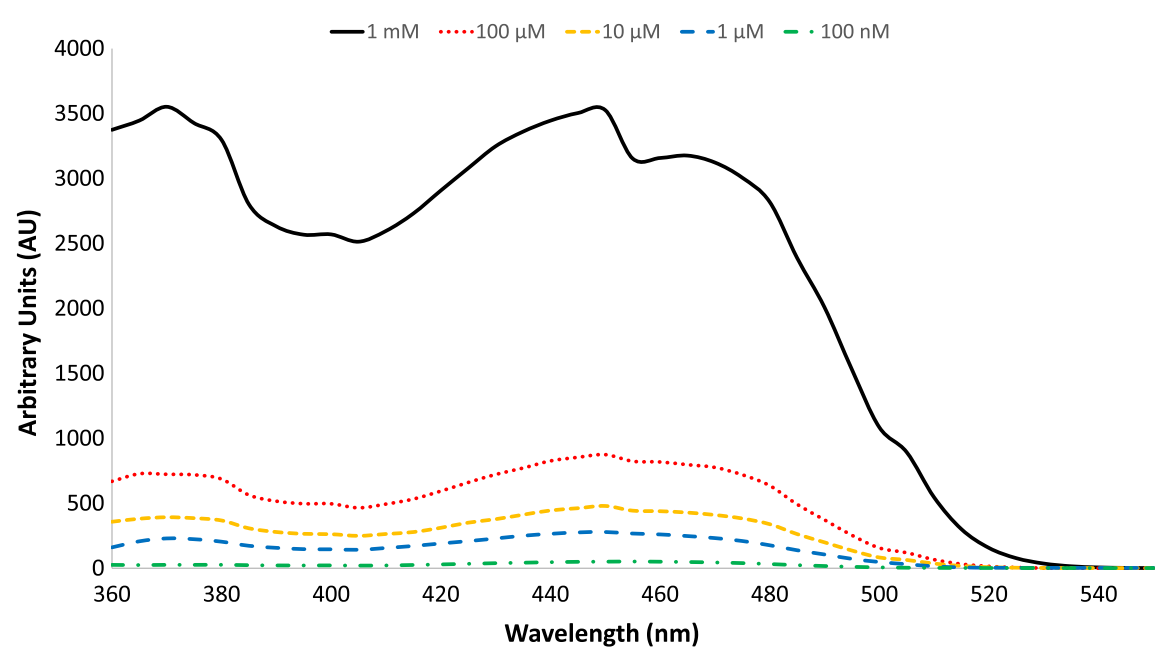

Fig. 1 A dilution response was completed for five concentrations of FAD. The initial $1 \mathrm{mM}$ concentration was created (black solid line) and repeatedly diluted to achieve concentrations of $100 \mu \mathrm{M}$ (red dotted line), $10 \mu \mathrm{M}$ (yellow short-dashed line), $1 \mu \mathrm{M}$ (blue long-dashed line), and $100 \mathrm{nM}$ (green dot-dashed line). Note that the intensity decreases as concentration decreases. 
a ratio was generated of signal per fluorophore in the neoplastic tissues compared with the corresponding normal specimens. A coefficient of variation (CV) was calculated for comparison among datasets as

$$
\mathrm{CV}=\frac{s}{\bar{x}} * 100 \%
$$

where $s$ is the standard deviation of the sample and $\bar{x}$ is the mean of the sample. This value was averaged across all wavelength bands to give a single $\mathrm{CV}$ for comparison among selected regions.

\section{Results and Discussion}

\subsection{Generation of Spectral Library}

To assess the relative contribution of each autofluorescent molecule to the bulk spectral signature of each specimen, we created an excitation-based spectral library of components known to be both autofluorescent and present in colorectal tissue. ${ }^{31-35}$ Excitation spectral data reported in the literature are frequently only reported in terms of peak excitation. ${ }^{31}$ However, using only the peak intensity wavelength does not provide sufficient information for identifying many molecular signatures in tissue. For example, elastin and NADH both have an excitation peak at $290 \mathrm{~nm}$, whereas collagen and elastin both have a peak at $325 \mathrm{~nm} .{ }^{31}$ To allow separation of signals from many autofluorescent molecules, we created a library of the excitation spectrum of each autofluorescent molecule between excitation wavelengths of 360 and $550 \mathrm{~nm}$ (Fig. 2), the same range used to acquire our tissue data. As the peak excitation wavelength for collagen and elastin fall near or below $360 \mathrm{~nm},{ }^{25,40-42}$ the measured spectra portray only the lagging portion of the spectrum for these two fluorophores. The same is true of NADH, with a much sharper cutoff of no additional excitation above $400 \mathrm{~nm}$. The peak for PPIX falls between 400 and $450 \mathrm{~nm}$, consistent with porphyrins in literature. ${ }^{31}$ The excitation peak wavelength of FAD was the longest peak wavelength at $450 \mathrm{~nm}$, also consistent with literature. ${ }^{31}$

\subsection{Spectral Mixture Validation}

To ensure the accuracy of our spectral library and test the efficacy of our LSU algorithm, the linear response of excitationscanning hyperspectral imaging was assessed using mixtures of two autofluorescent molecules in varying ratios. Pure autofluorescent molecule concentrations were first scanned to determine the range of linear intensity response as a function of concentration (Fig. 1) and construct the spectral library (Fig. 2). Based on these data, concentrations were selected for mixtures of autofluorescent molecules that provided a linear response and an equivalent intensity, as measured by the intensity at the peak wavelength. These equivalent intensity concentrations were then scaled to achieve ratio mixtures at which to assess the linearity of response of the excitation-scanning hyperspectral imaging and linear unmixing process. The response for FAD and NADH mixtures is shown as an example (Fig. 3). The following ratios of FAD to NADH were assessed: 100:0, 80:20, 60:40, 40:60, 20:80, and 0:100. The excitation-scanning hyperspectral imaging and linear unmixing process produced a linear response of detected abundance as a function of mixture composition. Here, $R^{2}$ values were 0.9685 and 0.9729 for FAD and $\mathrm{NADH}$, respectively. Unmixing error accounted for $<10 \%$ of the magnitude of the spectral signature measured. It is worth noting that while LSU represents a good or best-fit estimate, there is error associated with any fitting process, which may be exacerbated by additional fitting restrictions, such as nonnegative constraints. ${ }^{21}$ Furthermore, the electronic nature of spectral imaging systems is prone to some level of noise.

\subsection{Collection, Presentation, and Visualization of Spectral Image Data}

Fluorescence excitation image data were acquired from 1- to $2-\mathrm{cm}$ cubes of neoplastic and normal colon tissues using excitation wavelengths ranging from 360 to $550 \mathrm{~nm}$ in 5-nm increments. The resultant images were compiled into a hyperspectral image cube using a custom MATLAB script. Additionally, the intensity per pixel of each wavelength band in the image cube was summed across all wavelength bands to create a summed

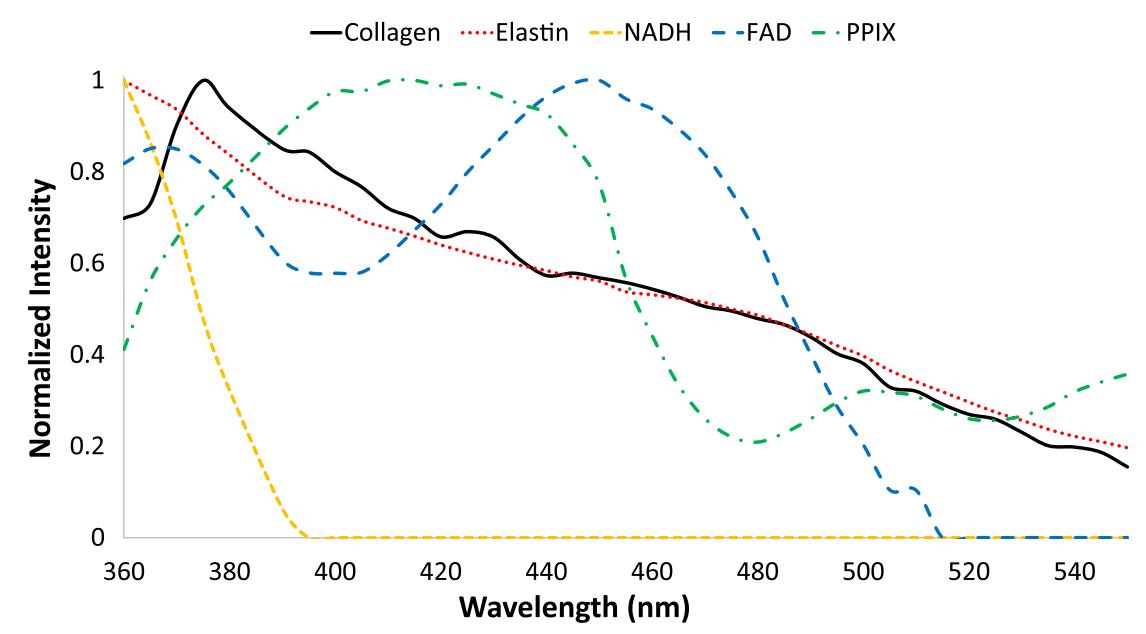

Fig. 2 The spectral library of pure endogenous fluorophores. Data were obtained by imaging sample suspensions of each component while ensuring that fluorophore concentrations were within the linear optical response range. The spectrum of each component was normalized to a value of unity at the wavelength with the strongest signal. Five fluorophores were selected: collagen (black solid line), elastin (red dotted line), FAD (blue long-dashed line), NADH (yellow short-dashed line), and PPIX (green dot-dashed line). 


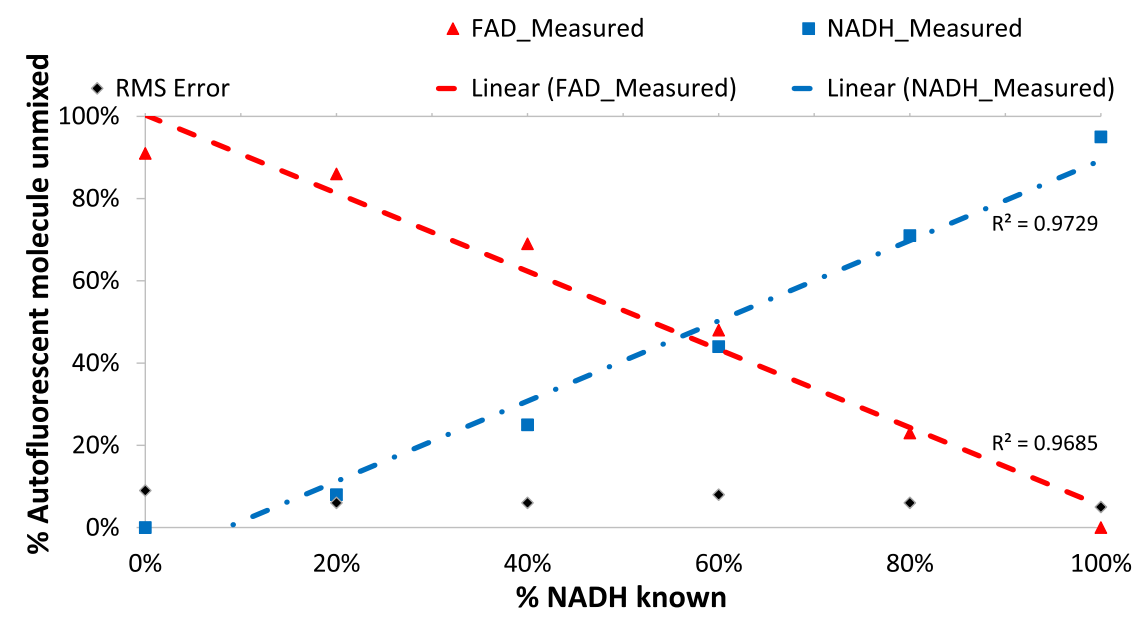

Fig. 3 The linearity of response of excitation-scanning hyperspectral imaging and linear unmixing was evaluated using mixtures of autofluorescent molecules, FAD and NADH. First, concentrations for FAD and $\mathrm{NADH}$ were determined that produced an equivalent intensity spectral response while ensuring linear optical interactions (10 $\mu \mathrm{M} \mathrm{FAD}$ and $100 \mu \mathrm{M} \mathrm{NADH})$. Based on these total concentrations, mixtures of $\mathrm{FAD}$ and $\mathrm{NADH}$ were prepared as a percent of the equivalent response concentration (e.g., 20\% FAD and $80 \%$ NADH). Mixtures were imaged using excitation-scanning spectral imaging and linearly unmixed. Unmixed abundances of FAD (red triangles) and NADH (blue squares) were plotted as a function of \% NADH in the prepared mixture. Linear fit of the abundances was also performed ( $F A D=$ red dashed line, $\mathrm{NADH}=$ blue dot-dashed line). RMS error is represented by black diamonds.

intensity image [Figs. 4(a)-4(d)]. For visualization, images were selected at set intervals throughout the scanning range, false colored [10\%, $375 \mathrm{~nm}$, blue, Fig. 4(e); 50\%, $455 \mathrm{~nm}$, green, Fig. 4(f); and 90\%, $530 \mathrm{~nm}$, red, Fig. 4(g)] and merged as an
RGB image [(Fig. 4(h)]. The false-colored image was used to select ROIs within each FOV [Fig. 4(i)]. The region-specific statistics were calculated as mean intensity and standard deviation intensity [Fig. 4(j)].
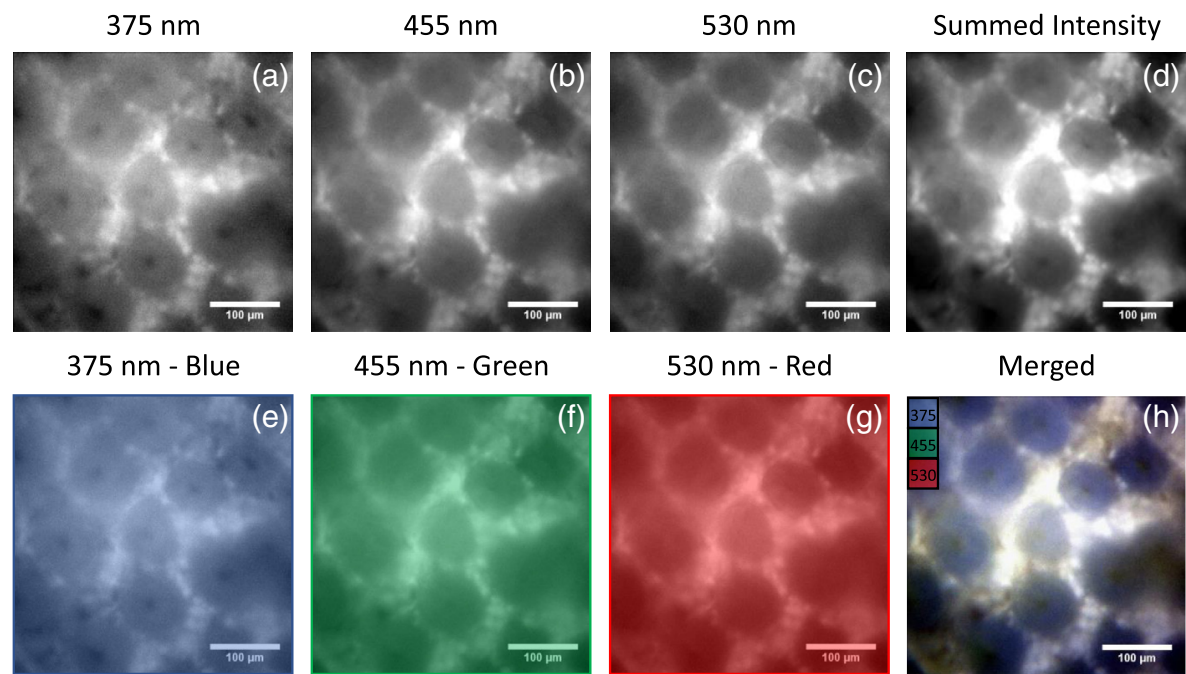

$455 \mathrm{~nm}$ - Green

$530 \mathrm{~nm}-\operatorname{Red}$

Merged
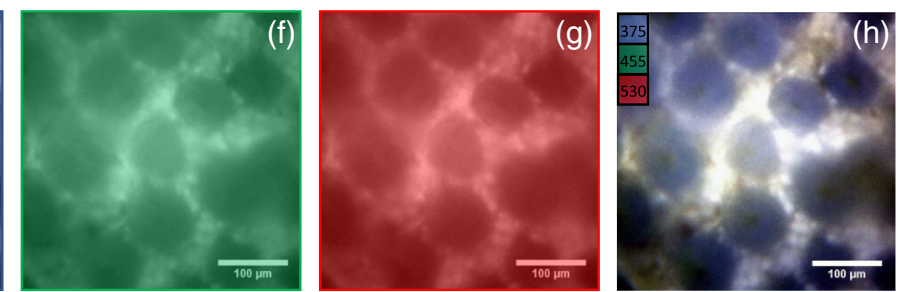

Selected ROI
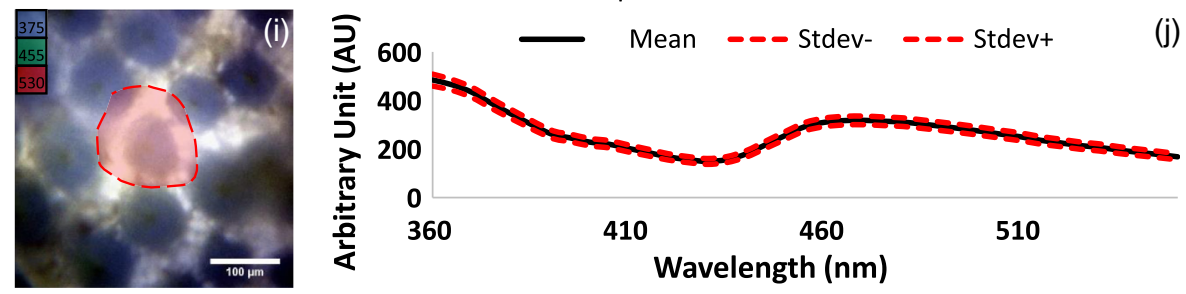

Fig. 4 Visualization of spectral image data. $(\mathrm{a}-\mathrm{c})$ Three single-band images and corresponding excitation wavelengths. (d) The image generated when the pixel intensity was summed for each wavelength band in the image cube. $(\mathrm{e}-\mathrm{g})$ The images in panels (a)-(c) with their respective false-color look-up tables applied. (h) The resultant image when (e)-(g) were merged. (i) An example region drawn on the merged image. (j) Region-specific statistics generated from the region shown in (i). The solid black line is the mean pixel intensity per wavelength and the dashed red lines are \pm 1 standard deviation. 


\subsection{Spectral Response per Region of Interest, Field of View, Specimen Type, and Patient}

Upon collecting a minimum of three FOVs per specimen type per patient, regions were drawn and region-specific data were examined. First, variability within the FOV was tested with multiple regions. In general, there was little variability in spectral shape within the FOV, regardless of where the region was drawn within normal colon tissue [Figs. 5(a)-5(e)]. Next, variability within the specimen type was tested by comparing spectral data over many FOVs per patient [Figs. 5(f)-5(j)]. The variability across multiple FOVs was higher than in selected
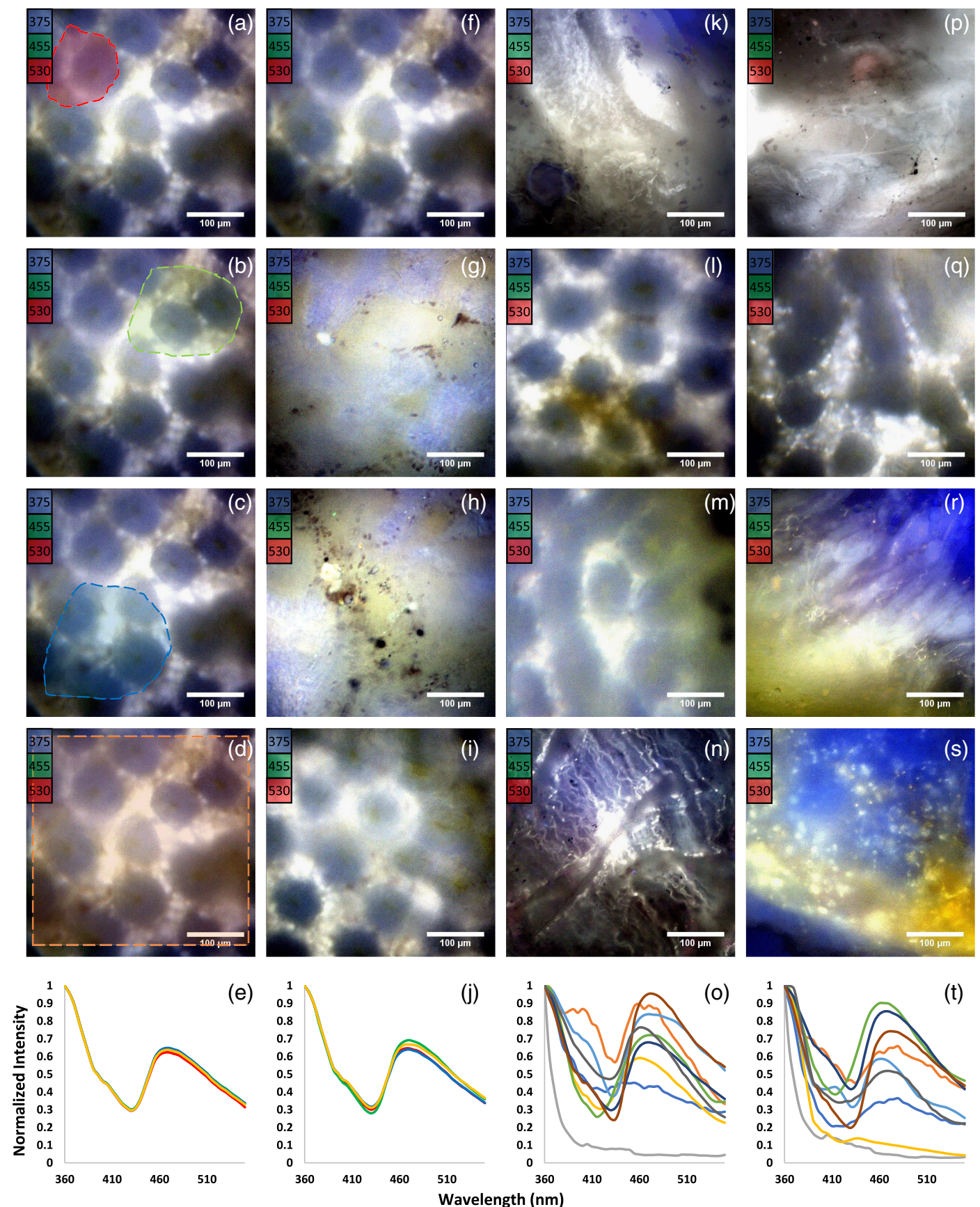

Fig. 5 Spectral characteristics of image data. (a)-(d) A representative false-colored normal colon specimen image and an overlaid region (shown by dashed line) with varying sizes and locations selected to examine for spectral variability within the image. (e) Color-coded plot of the selected regions where the color of each line in the plot presents the average signature within the correspondingly colored region in panels (a)-(d). (f)-(i) Four different FOVs of the same normal colon specimen selected to determine spectral variability within the representative specimen. (j) Average spectral response of (f) (red), (g) (green), (h) (blue), and (i) (orange). (k)-(n) Example FOVs from normal specimens from four different patients. (o) Average normal colon specimen spectral response of each patient in the study. (p)(s) Representative FOVs from neoplastic specimens from four different patients. (t) Average neoplastic spectral response of each patient in the study. 


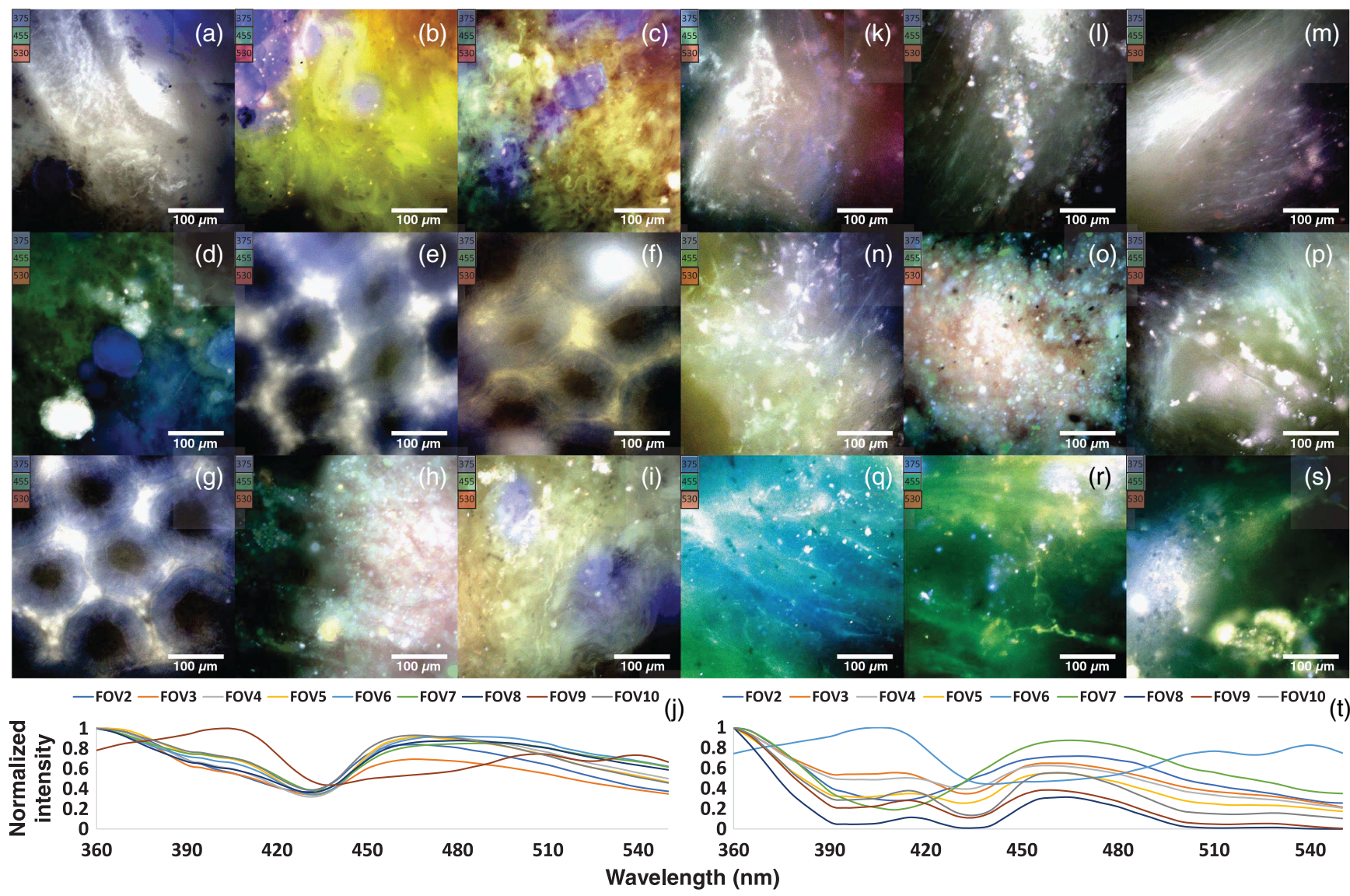

Fig. 6 Eighteen FOVs from a single patient and their respective pixel-averaged spectra. (a)-(i) FOVs 2 to 10 of the normal colon section of patient 5. (FOV1 was omitted as it was used to collect a background spectrum.) (j) The plotted pixel-averaged spectra of each FOV shown in (a)-(i). (k)-(s) FOVs 2 to 10 of the neoplastic section of patient 5. (FOV1 was omitted as it was used to collect a background spectrum.) (t) The plotted pixel-averaged spectra of each FOV shown in (k)-(s).

regions of a single FOV [CV of $1 \%$ versus $\mathrm{CV}$ of $4 \%$, respectively, Figs. 5(a)-5(j)]. These data were used to characterize an overall average spectrum per patient for each neoplasm and normal colon tissue specimen. While normal colon [Figs. 5(k)5(o)] and neoplastic specimens [Figs. 5(p)-5(t)] shared some of the same spectral characteristics (e.g., similar peak locations), the normal colon spectra were less variant between patients (38\% CV versus $49 \%$ for neoplastic specimens). This suggests that the overall spectral response in normal colon tissue has a more defined signature whereas neoplastic specimens differ from this signature with increased variability, possibly due to variation in changes of cellular and extracellular matrix (ECM) composition associated with remodeling of the tumor environment. ${ }^{32,33,35,43}$ Variation from a defined signature was more evident when data from normal colon specimens and neoplastic specimens were compared within a single patient (Fig. 6). To mitigate error due to enhanced spectral variation within neoplastic specimens and to better approximate bulk properties of the specimen, a higher number of FOVs were acquired for neoplastic specimens that presented noticeable within-field variability and each entire FOV was selected for the pixel-averaged ROI.

\subsection{Unmixing Images by Library Components}

Neoplastic processes can alter the ECM comopsition. ${ }^{44-47}$ Although hematoxylin and eosin (H\&E) staining is the standard diagnostic method for colorectal neoplasms, the desmoplastic response, or change in matrix materials such as collagen and elastin, also aids pathologists in the interpretation of neoplastic processes, particularly regarding the presence or absence of invasion in colorectal adenocarcinoma. ${ }^{34,48}$ Additionally, disruption of the metabolic cycle in neoplastic cells can result in changes in FAD and NADH concentrations. ${ }^{32,33,35,49}$ Estimating the relative contributions of collagen, elastin, FAD, and $\mathrm{NADH}$ in neoplastic and normal tissues may provide a mechanism for detecting cancer-related changes in the ECM. ${ }^{50-52}$ LSU was used to estimate relative abundances of autofluorescent molecules, allowing estimation of localized fluorophore relative concentration (Fig. 7). Elastin and NADH were often responsible for most of the fluorescence intensity in each FOV. Collagen was regularly the third most prevalent contributor, and typically in localized, concentrated regions. FAD generally had a low abundance and uniform distribution. PPIX usually comprised no more than $3 \%$ of the total fluorescence intensity. (Raw data are shown in Table 1.)

\subsection{Comparison of Normal Colon and Neoplastic Fluorescence Abundances}

This investigation sought to determine whether estimated abundances of autofluorescent molecules could be used as a diagnostic tool to indicate colorectal neoplasia. A repeatable 
Deal et al.: Identifying molecular contributors to autofluorescence of neoplastic and normal colon sections using excitation-scanning...

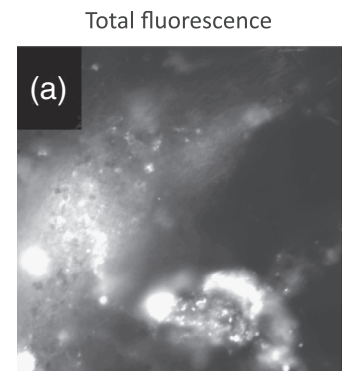

Unmixed FAD

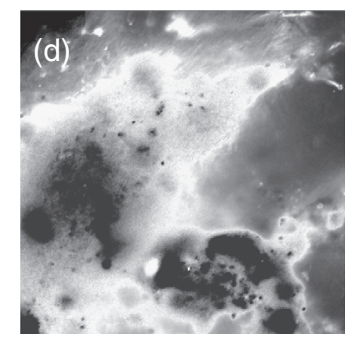

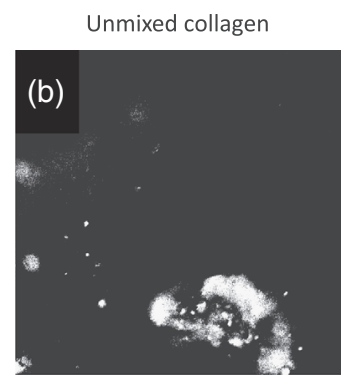

Unmixed NADH

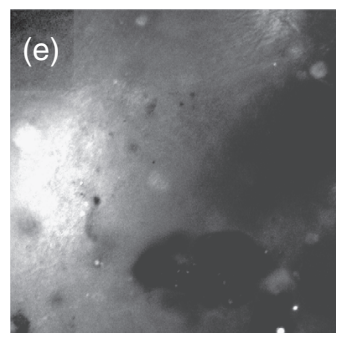

Unmixed elastin

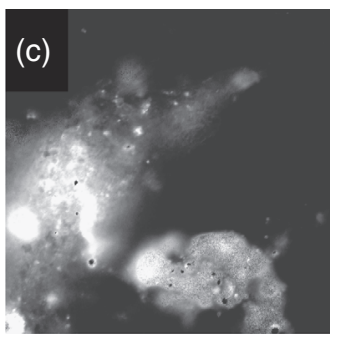

Unmixed PPIX

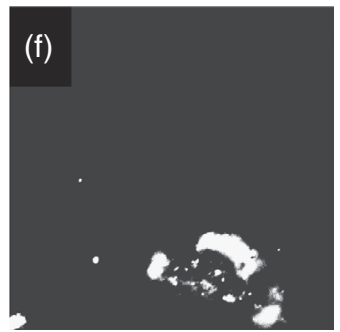

Fig. 7 The relative signal contributions from each purified fluorescent molecule were estimated using LSU and a corresponding excitation-scanning spectral imaging library. (a) The summed fluorescence signal created by adding the intensity at each pixel per image in the hyperspectral image cube. (b)-(f) The unmixed abundance image for each fluorophore. Images were linearly scaled for visualization purposes only between the range of the minimum and maximum intensity per image.

Table 1 The raw unmixed data for each fluorophore for each tissue type and patient. Note that many of the ratios of collagen, FAD, and PPIX are not calculable, as one or both of the pairs is 0 .

\begin{tabular}{|c|c|c|c|c|c|c|}
\hline & Collagen & Elastin & FAD & $\mathrm{NADH}$ & PPIX & RMS error \\
\hline HSI1 normal colon & 0.00 & 430.41 & 0.00 & 105.21 & 0.00 & 110.16 \\
\hline HSI1 neoplastic colon & 1.90 & 149.22 & 0.85 & 184.17 & 0.00 & 68.50 \\
\hline HSI2 normal colon & 867.96 & 4600.26 & 271.98 & 59.96 & 158.41 & 1449.29 \\
\hline HSI2 neoplastic colon & 7.70 & 2301.56 & 0.00 & 95.61 & 68.27 & 1040.85 \\
\hline HSI3 normal colon & 0.00 & 223.56 & 0.00 & 1869.46 & 63.68 & 400.15 \\
\hline HSI3 neoplastic colon & 0.00 & 79.83 & 9.27 & 1210.98 & 142.39 & 236.35 \\
\hline HSI4 normal colon & 31.97 & 103.31 & 13.67 & 238.23 & 0.00 & 90.34 \\
\hline HSI4 neoplastic colon & 69.34 & 83.34 & 1.70 & 518.24 & 0.40 & 60.04 \\
\hline HSI5 normal colon & 14.21 & 426.62 & 0.00 & 10.30 & 14.33 & 281.88 \\
\hline HSI5 neoplastic colon & 3.90 & 183.03 & 23.55 & 47.43 & 29.65 & 142.55 \\
\hline HSI6 normal colon & 40.32 & 116.14 & 6.74 & 114.75 & 0.00 & 70.81 \\
\hline HSI6 neoplastic colon & 0.00 & 109.85 & 9.18 & 0.06 & 0.00 & 80.83 \\
\hline HSI7 normal colon & 0.00 & 377.21 & 0.00 & 35.47 & 0.00 & 214.68 \\
\hline HSI7 neoplastic colon & 0.44 & 352.29 & 1.50 & 11.06 & 0.00 & 152.61 \\
\hline HSI8 normal colon & 0.02 & 42.76 & 0.00 & 1.27 & 0.00 & 38.62 \\
\hline HSI8 neoplastic colon & 0.00 & 187.93 & 0.00 & 24.55 & 0.00 & 151.59 \\
\hline HSI9 normal colon & 0.11 & 150.62 & 11.62 & 6.73 & 0.00 & 64.85 \\
\hline HSI9 neoplastic colon & 72.19 & 31.75 & 2.55 & 161.02 & 2.13 & 29.09 \\
\hline
\end{tabular}


(a)

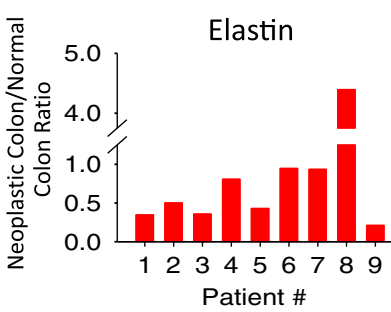

(b)

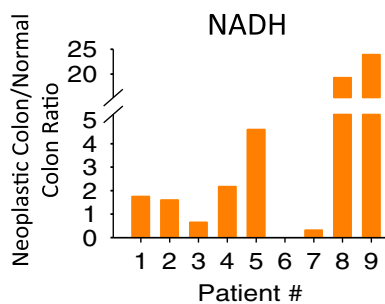

(c)

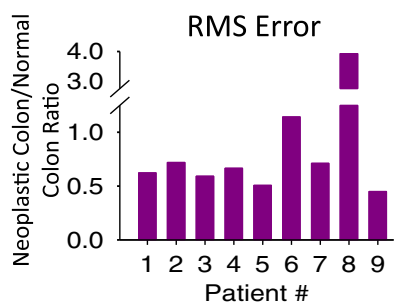

Fig. 8 The ratio of neoplastic-to-normal colon average abundance values for each patient for elastin and $\mathrm{NADH}$. The ratio was computed as (neoplastic abundance/normal colon abundance). Collagen, FAD, and PPIX ratios were not meaningful due to near 0 signal in most unmixed images. (a) The ratio of neoplastic-to-normal colon signal for elastin was $<1$ for 8 of the 9 patients, indicating more fluorescence due to elastin in normal colon specimens than neoplastic specimens. (b) The ratio of neoplastic-to-normal colon signal for $\mathrm{NADH}$ was $>1$ for 6 of the 9 patients, indicating less fluorescence due to NADH in normal colon specimens than neoplastic specimens. (c) The ratio of residual fluorescence unable to be accounted for by the spectral library $<1$ for 7 of 9 patients, indicating a more encompassing unmixing for the neoplastic specimens than the normal colon specimens.

spectral deviation in tissue signature should indicate a repeatable change in tissue composition that may be used as a marker for colorectal cancer and its precursors. To test this theory, ImageJ was used to extract region-specific spectral statistics for each unmixed image. These measurements were averaged per tissue specimen to compare normal colon tissues with their neoplastic counterparts. This comparison was performed by calculating the ratio of total fluorescence intensity in each unmixed abundance image of the neoplastic specimen divided by the intensity of the corresponding normal colon specimen (Fig. 8). Collagen, FAD, and PPIX ratios were not meaningful, as many patients had near zero fluorescence contribution in either the neoplastic or normal colon specimens. (Raw data are shown in Table 1.) The elastin ratio was below 1 for 8 of the 9 patients. This may indicate an elastin decrease in neoplastic specimens due to ECM remodeling, possibly by breakdown of elastin through neoplasticderived factors. ${ }^{53-55}$ Furthermore, the NADH ratio was above 1 for 6 of the 9 patients, possibly indicating increased metabolic activity in neoplastic regions. Additionally, RMS error decreased in neoplastic specimens, indicating a better fit of the autofluorescent molecule library for the neoplastic specimens than their normal colon counterparts. One possible explanation is that there are molecules not accounted for in our autofluorescence library that are usually present in normal colon tissue and are degraded in neoplastic conditions, thus reducing the number of fluorophores needed in the spectral library to account for the spectrum of neoplastic tissues. ${ }^{31,53}$

\subsection{Limitations}

There are a few important points to note about this study. (1) The current sample size is 9 normal and neoplastic colon tissue pairs. Continued sample accumulation is expected to reveal trends not discernible within the current sample size. (2) In addition, to maximize the number of samples available for the study, data were pooled into two broad categories: normal colon and neoplastic colon, regardless of whether the neoplasm represented colon adenocarcinoma or a precursor lesion. Data acquired in this study represent a range of colorectal neoplasm types, including adenomatous polyps and invasive adenocarcinoma. Hence, one limitation may be that normal colon specimens could have been isolated from different layers of the colon (as opposed to, for example, only the mucosal layer) and that neoplastic colon specimens could represent invasive adenocarcinoma or an adenomatous polyp, as noted previously. Furthermore, tissue collected as normal colon from patients under treatment for colorectal cancer may differ from that of a patient devoid of neoplasms. (3) The collagen and elastin library spectral signatures in this study were similar and difficult to separate with LSU. Additionally, the quantum yield of NADH is low for wavelengths $>400 \mathrm{~nm}$, whereas collagen and elastin have peak excitation wavelengths in the ultraviolet region $(<400 \mathrm{~nm})$. Thus, estimates of abundance of these molecules would be more accurate with an extended wavelength scanning range. (4) This LSU process used a spectral library containing five fluorophores, which assumes the existence of a complete spectral library. That is, some pixels may have been inappropriately labeled as one of the five fluorophores, when in fact, they belong to a missing additional fluorophore. As the amount of signal present in the RMS error accounted for more abundance than some of the spectral library components, an additional autofluorescent molecule is likely unaccounted for. Interestingly, the signal due to RMS error was lower in neoplastic specimens. This could indicate a breakdown within the neoplastic specimens of the autofluorescent components not accounted for in our spectral library, ${ }^{53-55}$ resulting in a better fit. Future work will assess the ability of nonlinear unmixing and other spectral analysis methods, which utilize similarity or spectral distance metrics and do not require a complete library, to estimate autofluorescent molecule abundances. In an ideal experiment, every source of fluorescence would be identified and its abundance quantified. However, for practical purposes, many pathologies may be diagnosable using only a limited number of autofluorescent molecules in the spectral library. (5) This study only considered a single type of each fluorescent molecule (e.g., collagen I for collagen). Previous fluorescence imaging studies have shown spectral differences among the various types of collagen. ${ }^{56-58}$ It is reasonable to assume that different collagen types could have distinct spectral signatures when measured with excitation-scanning hyperspectral imaging. In future work, we plan to account for a wider range of autofluorescent molecules, including other types of collagen.

\section{Conclusions and Future Work}

Differentiation of neoplastic and normal colon tissues remains a challenge for the detection and diagnosis of colorectal cancer and its precursors. Development of an imaging system for quick, sensitive, and accurate differentiation of colorectal tissues 
would serve both clinicians and patients. In this study, we examined the possibility of using fluorescence excitation signals from endogenous fluorophores to increase sensitivity and accuracy of colorectal neoplasm detection. The data presented here represent the basis for a study of the contribution of individual autofluorescent molecules to overall tissue fluorescence in normal colon and neoplastic colorectal tissues. Though some molecules appear to remain unchanged in disease states, there is a shift in the contribution of several autofluorescent molecules from normal to neoplastic measurements. The shifts caused by the changing ratios of these molecules will affect the overall shape of the spectral response. Hence, the diagnostic potential may lie in algorithms that accurately identify when the spectrum of an abnormal tissue deviates from the known spectrum of normal tissue. These algorithms can be used to give a more intuitive, visually obvious metric for noticing a shift from normal colon to neoplastic tissue. We plan to evaluate histogram distance metrics as a means for identifying deviations in the spectral shape of a specimen from the spectral shape of normal tissue.

\section{Disclosures}

Drs. Leavesley and Rich disclose financial interest in a start-up company formed to commercialize spectral imaging technologies, SpectraCyte, LLC.

\section{Acknowledgments}

We would like to acknowledge the Abraham Mitchell Cancer Research fund, the National Science Foundation under Grant No. 1725937, the American Heart Association under Grant No. 18PRE34060163, and the National Institutes of Health under Grant Nos. P01 HL 066299, UL1 TR001417, S10 RR027535, and S10 OD020149 for supporting this work. This work was previously presented in parts of several Photonics West conferences. ${ }^{59-67}$

\section{References}

1. A. H. Coons et al., "The demonstration of pneumococcal antigen in tissues by the use of fluorescent antibody," J. Immunol. 45(3), 159-170 (1942).

2. D. C. Prasher et al., "Primary structure of the Aequorea victoria greenfluorescent protein," Gene 111(2), 229-233 (1992).

3. R. Heim and R. Y. Tsien, "Engineering green fluorescent protein for improved brightness, longer wavelengths and fluorescence resonance energy transfer," Curr. Biol. 6(2), 178-182 (1996).

4. M. Chalfie et al., "Green fluorescent protein as a marker for gene expression," Science 263, 802-805 (1994).

5. H. Kobayashi et al., "New strategies for fluorescent probe design in medical diagnostic imaging," Chem. Rev. 110(5), 2620-2640 (2009).

6. J. C. Waters, "Accuracy and precision in quantitative fluorescence microscopy," J. Cell Biol. 185(7), 1135 (2009).

7. J. T. Alander et al., "A review of indocyanine green fluorescent imaging in surgery," J. Biomed. Imaging 2012, 940585 (2012).

8. R. A. Schultz et al., "Hyperspectral imaging: a novel approach for microscopic analysis," Cytometry A 43(4), 239-247 (2001).

9. A. F. H. Goetz, "Three decades of hyperspectral remote sensing of the Earth: a personal view," Remote Sens. Environ. 113, S5-S16 (2009).

10. A. F. Goetz, "Measuring the Earth from above: 30 years (and counting) of hyperspectral imaging," Photonics Spectra 45(6), 42-47 (2011).

11. R. Lu and Y.-R. Chen, "Hyperspectral imaging for safety inspection of food and agricultural products," Proc. SPIE 3544, 121-133.

12. H. Liang, "Advances in multispectral and hyperspectral imaging for archaeology and art conservation," Appl. Phys. A 106(2), 309-323 (2012).

13. E. K. Hege et al., "Hyperspectral imaging for astronomy and space surveillance," Proc. SPIE 5159, 380-392 (2004).
14. T. Vo-Dinh, "A hyperspectral imaging system for in vivo optical diagnostics," IEEE Eng. Med. Biol. Mag. 23(5), 40-49 (2004).

15. G. Edelman, T. G. van Leeuwen, and M. C. Aalders, "Hyperspectral imaging for the age estimation of blood stains at the crime scene," Forensic Sci. Int. 223(1), 72-77 (2012).

16. G. A. Swayze et al., "Using imaging spectroscopy to map acidic mine waste," Environ. Sci. Technol. 34(1), 47-54 (2000).

17. B. Khoobehi, J. M. Beach, and H. Kawano, "Hyperspectral imaging for measurement of oxygen saturation in the optic nerve head," Invest. Ophthalmol. Vis. Sci. 45(5), 1464-1472 (2004).

18. A. Gowen et al., "Hyperspectral imaging_an emerging process analytical tool for food quality and safety control," Trends Food Sci. Technol. 18(12), 590-598 (2007).

19. G. Edelman et al., "Hyperspectral imaging for non-contact analysis of forensic traces," Forensic Sci. Int. 223(1), 28-39 (2012).

20. P. W. Yuen and M. Richardson, "An introduction to hyperspectral imaging and its application for security, surveillance and target acquisition," Imaging Sci. J. 58(5), 241-253 (2010).

21. P. Ghamisi et al., "Advances in hyperspectral image and signal processing: a comprehensive overview of the state of the art," IEEE Geosci. Remote Sens. Mag. 5(4), 37-78 (2017).

22. R. Alfano et al., "Optical spectroscopic diagnosis of cancer and normal breast tissues," J. Opt. Soc. Am. B 6(5), 1015-1023 (1989).

23. V. V. Tuchin, S. R. Utz, and I. V. Yaroslavsky, "Tissue optics, light distribution, and spectroscopy," Opt. Eng. 33(10), 3178-3189 (1994).

24. G. A. Wagnieres, W. M. Star, and B. C. Wilson, "In vivo fluorescence spectroscopy and imaging for oncological applications," Photochem. Photobiol. 68(5), 603-632 (1998).

25. R. Richards-Kortum and E. Sevick-Muraca, "Quantitative optical spectroscopy for tissue diagnosis," Annu. Rev. Phys. Chem. 47(1), 555-606 (1996).

26. S. Leavesley et al., "An excitation wavelength-scanning spectral imaging system for preclinical imaging," Rev. Sci. Instrum. 79(2), 023707 (2008).

27. P. Favreau et al., "Thin-film tunable filters for hyperspectral fluorescence microscopy," J. Biomed. Opt. 19(1), 011017 (2014).

28. P. F. Favreau et al., "Excitation-scanning hyperspectral imaging microscope," J. Biomed. Opt. 19(4), 046010 (2014).

29. American Cancer Society, "Colorectal cancer," https://www.cancer.org/ cancer/colon-rectal-cancer.html (10 September 2018).

30. R. L. Siegel, K. D. Miller, and A. Jemal, "Colorectal cancer mortality rates in adults aged 20 to 54 years in the United States, 1970-2014," J. Am. Med. Assoc. 318(6), 572-574 (2017).

31. N. Ramanujam, "Fluorescence spectroscopy of neoplastic and non-neoplastic tissues," Neoplasia 2(1-2), 89-117 (2000).

32. Y. S. Korneva, A. E. Dorosevich, and V. S. Maryakhina, "Fluorescent diagnostics of epithelial neoplasms of different colon parts," Lasers Surg. Med. 49(8), 763-766 (2017).

33. Y. S. Korneva, A. Dorosevich, and V. Maryakhina, "Investigation of malignant transformation of sporadic adenomas of colon by fluorimetry," Lasers Med. Sci. 32(2), 363-367 (2017).

34. M. Nebuloni et al., "Insight on colorectal carcinoma infiltration by studying perilesional extracellular matrix," Sci. Rep. 6, 22522 (2016).

35. B. Banerjee et al., "Enhanced visibility of colonic neoplasms using formulaic ratio imaging of native fluorescence," Lasers Surg. Med. 45(9), 573-581 (2013).

36. S. J. Leavesley et al., "Hyperspectral imaging microscopy for identification and quantitative analysis of fluorescently-labeled cells in highly autofluorescent tissue," J. Biophotonics 5(1), 67-84 (2012).

37. N. Annamdevula et al., "An approach for characterizing and comparing hyperspectral microscopy systems," Sensors 13(7), 9267-9293 (2013).

38. A. R. Williams, An Introduction to Fluorescence Spectroscopy, PerkinElmer, Beaconsfield, Buckinghamshire, United Kingdom (1981).

39. Y. Yang and D. Zhang, "Concentration effect on the fluorescence spectra of humic substances," Commun. Soil Sci. Plant Anal. 26(15-16), 2333-2349 (1995).

40. S. Bagdonas et al., "Spectroscopic studies of the human heart conduction system ex vivo: implication for optical visualization," J. Photochem. Photobiol. B 92(2), 128-134 (2008).

41. K. T. Schomacker et al., "Ultraviolet laser-induced fluorescence of colonic tissue: basic biology and diagnostic potential," Lasers Surg. Med. 12(1), 63-78 (1992). 
42. B.-H. Li and S.-S. Xie, "Autofluorescence excitation-emission matrices for diagnosis of colonic cancer," World J. Gastroenterol. 11(25), 3931 (2005).

43. J. Yi et al., "Spatially resolved optical and ultrastructural properties of colorectal and pancreatic field carcinogenesis observed by inverse spectroscopic optical coherence tomography," J. Biomed. Opt. 19(3), 036013 (2014).

44. A. Afasizheva et al., "Mitogen-activated protein kinase signaling causes malignant melanoma cells to differentially alter extracellular matrix biosynthesis to promote cell survival," BMC Cancer 16(1), 186 (2016).

45. B. Krishnamachary et al., "Breast cancer cell cyclooxygenase-2 expression alters extracellular matrix structure and function and numbers of cancer associated fibroblasts," Oncotarget 8(11), 17981 (2017).

46. K. Vaiphei, "Molecular interphase between extracellular matrix \& cancer cells," Indian J. Med. Res. 146(3), 298-300 (2017).

47. M. Ward and J. Spiers, "Protein phosphatase 2A regulation of markers of extracellular matrix remodelling in hepatocellular carcinoma cells: functional consequences for tumour invasion," $\mathrm{Br}$. J. Pharmacol. 174(10), 1116-1130 (2017).

48. C. Hwang et al., "Venous invasion in colorectal cancer: impact of morphologic findings on detection rate," Cancer Res. Treat. 48(4), 1222-1228 (2016).

49. X. Cui et al., "High expression of NQO1 is associated with poor prognosis in serous ovarian carcinoma," BMC Cancer 15(1), 244 (2015).

50. J. H. Ostrander et al., "Optical redox ratio differentiates breast cancer cell lines based on estrogen receptor status," Cancer Res. 70(11), 4759_ 4766 (2010).

51. S. Palmer et al., "Optical redox ratio and endogenous porphyrins in the detection of urinary bladder cancer: a patient biopsy analysis," J. Biophotonics 10(8), 1062-1073 (2017).

52. Q. Liu et al., "Compact point-detection fluorescence spectroscopy system for quantifying intrinsic fluorescence redox ratio in brain cancer diagnostics," J. Biomed. Opt. 16(3), 037004 (2011).

53. A. H. Said, J.-P. Raufman, and G. Xie, "The role of matrix metalloproteinases in colorectal cancer," Cancers 6(1), 366-375 (2014).

54. S. R. Van Doren, "Matrix metalloproteinase interactions with collagen and elastin," Matrix Biol. 44, 224-231 (2015).

55. A. C. Lau et al., "Matrix metalloproteinase 9 activity leads to elastin breakdown in an animal model of Kawasaki disease," Arthritis Rheum. Off. J. Am. Coll. Rheumatol. 58(3), 854-863 (2008).

56. K. Belbachir et al., "Collagen types analysis and differentiation by FTIR spectroscopy," Anal. Bioanal. Chem. 395(3), 829-837 (2009).

57. T. Nguyen et al., "Characterization of type I and IV collagens by Raman microspectroscopy: identification of spectral markers of the dermoepidermal junction," J. Spectrosc. 27(5-6), 421-427 (2012).

58. S. Ranjit et al., "Imaging fibrosis and separating collagens using second harmonic generation and phasor approach to fluorescence lifetime imaging," Sci. Rep. 5, 13378 (2015).

59. S. J. Leavesley et al., "Hyperspectral imaging fluorescence excitation scanning for detecting colorectal cancer: pilot study," Proc. SPIE 9703, 970315 (2016).

60. S. A. Mayes, S. J. Leavesley, and T. C. Rich, "Excitation-scanning hyperspectral imaging system for microscopic and endoscopic applications," Proc. SPIE 9711, $97110 \mathrm{Z}$ (2016).

61. P. F. Favreau et al., "Feasibility for detection of autofluorescent signatures in rat organs using a novel excitation-scanning hyperspectral imaging system," Proc. SPIE 9711, 971113 (2016).

62. S. J. Leavesley et al., "Comparing methods for analysis of biomedical hyperspectral image data," Proc. SPIE 10068, 100680S (2017).

63. S. A. Mayes et al., "Optimization and applications of an excitation-scanning hyperspectral imaging system," Proc. SPIE 10076, 1007609 (2017).

64. J. Deal et al., "Excitation-scanning hyperspectral imaging as a means to discriminate various tissues types," Proc. SPIE 10068, 1006816 (2017).
65. S. J. Leavesley et al., "Colorectal cancer detection by hyperspectral imaging using fluorescence excitation scanning," Proc. SPIE 10489, 104890K (2018).

66. S. A. Mayes et al., "Applications and assessment of an excitationscanning hyperspectral imaging system," Proc. SPIE 10497, 1049706 (2018).

67. J. Deal et al., "Demystifying autofluorescence with excitation scanning hyperspectral imaging," Proc. SPIE 10497, 1049715 (2018).

Joshua Deal received his BS degree in mathematics from Troy University in 2011 and his MS degree in mathematics from the University of South Alabama in 2013. Currently, he is pursuing a $\mathrm{PhD}$ in basic medical sciences at the University of South Alabama. His research interests include applications of spectral imaging technologies and advanced data analysis approaches for research, biomedical, and clinical applications.

Sam Mayes recived his BS and MS degrees in chemical and biomolecular engineering in 2014 and 2017, and he earned his DSc degree in systems engineering in 2018 from the University of South Alabama. His research interests include hyperspectral imaging, machine learning, and chemical detection. Currently, he is working as a contract engineer.

Craig Browning received his BSc and MSc degrees in chemical and biomolecular engineering from the University of South Alabama in 2016 and 2018, respectively. Currently, he is pursuing a DSc degree in systems engineering at the University of South Alabama. His research interests include integrating spectral imaging techniques in endoscopy and other clinical applications.

Shante Hill: Biography is not available.

Paul Rider is an associate professor in the Department of Surgery, and chief of the Division of Colorectal Surgery. He also serves as an associate program director of the surgery residency program. $\mathrm{He}$ is board certified in both general and colorectal surgery. He is a fellow in the American College of Surgeons and the American Society of Colorectal Surgery. He is actively engaged in medical research and community service.

Carole Boudreaux graduated from the Louisiana State University School of Medicine in Shreveport, Louisiana, in 1990. She completed a combined anatomic and clinical pathology residency at the University of South Alabama in Mobile, Alabama, in 1995. She is certified in anatomic and clinical pathology by the American Board of Pathology with added certification in cytopathology. Currently, she is an associate professor in the Department of Pathology at the University of South Alabama.

Thomas C. Rich received his BAE and MS degrees in aerospace engineering from the Georgia Institute of Technology in 1988 and 1990, and his $\mathrm{PhD}$ degrees in biomedical engineering from Vanderbilt University in 1996. Currently, he is a professor in the Department of Pharmacology and Center of Lung Biology at the University of South Alabama. His research interests include the implementation of spectral imaging approaches for both basic and translational research.

Silas J. Leavesley received his BSc degree in chemical engineering in 2003 from Florida State University and his PhD in biomedical engineering in 2008 from Purdue University. Currently, he is a professor in the Departments of Chemical and Biomolecular Engineering, Pharmacology, and the Center for Lung Biology at the University of South Alabama. His research interests lie in the development of spectral imaging technologies for medical sciences research and clinical applications. 